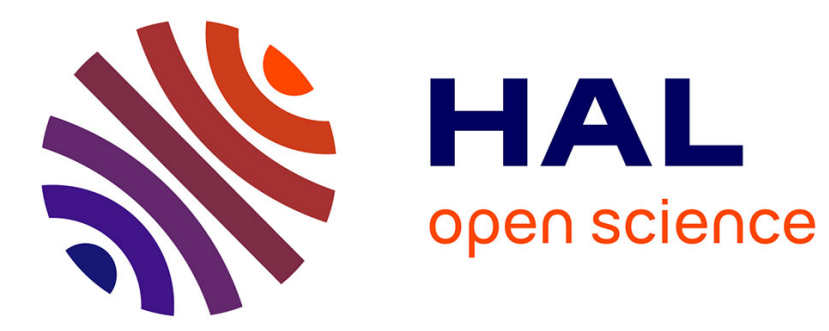

\title{
Canope et les orientations nord-sud de Karnak établies par Thoutmosis III
}

Luc Gabolde

\section{To cite this version:}

Luc Gabolde. Canope et les orientations nord-sud de Karnak établies par Thoutmosis III. Revue d'egyptologie, 1999. hal-01895030

\section{HAL Id: hal-01895030 \\ https://hal.science/hal-01895030}

Submitted on 15 Oct 2018

HAL is a multi-disciplinary open access archive for the deposit and dissemination of scientific research documents, whether they are published or not. The documents may come from teaching and research institutions in France or abroad, or from public or private research centers.
L'archive ouverte pluridisciplinaire HAL, est destinée au dépôt et à la diffusion de documents scientifiques de niveau recherche, publiés ou non, émanant des établissements d'enseignement et de recherche français ou étrangers, des laboratoires publics ou privés. 


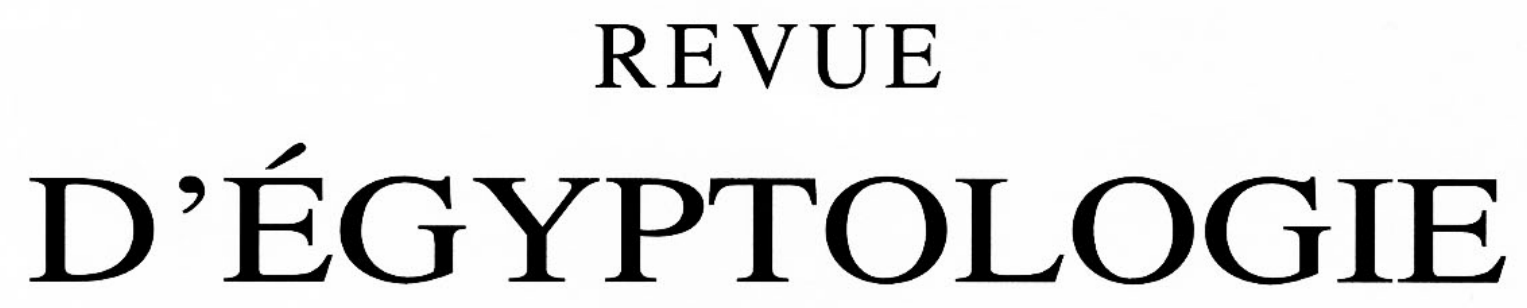

PUBLIÉE PAR

LA SOCIÉTÉ FRANÇAISE D’ÉGYPTOLOGIE

AVEC LE CONCOURS DU CENTRE NATIONAL DE LA RECHERCHE SCIENTIFIQUE

TOME 50

PARIS

ÉDITIONS PEETERS

1999 


\section{CANOPE ET LES ORIENTATIONS NORD-SUd DE KARNAK ÉTABLIES PAR THOUTMOSIS III}

Parmi les rois qui ont œuvré dans le temple d'Amon-Rê, il en est trois dont les interventions conditionnèrent le plan d'aménagement du temple: Antef II parce qu'il est à ce jour le plus ancien à avoir bâti pour Amon sur le site, Sésostris I ${ }^{\text {er }}$ qui reconstruisit intégralement le temple et Thoutmosis III (en corégence avec Hatchepsout ou seul) qui transposa le culte dans l'Akhmenou et jalonna l'axe sud des $\mathrm{VIII}^{e}$ et $\mathrm{VIII}^{\mathrm{e}}$ pylônes ${ }^{1}$. Les tracés directeurs qu'ils implantèrent fixèrent pour l'avenir des axes dont les azimuts (l'angle qu'ils forment par rapport au nord géographique) furent apparemment déterminés avec soin par des visées astrales.

\section{Axes nord-sud de Thoutmosis III}

L'axe majeur du grand temple d'Amon-Rê à Karnak a été tracé lors de la refondation du sanctuaire par Sésostris Ir. Il est orienté est-ouest (azimut: $116^{\circ} \mathbf{4 3}$ ' 7,35") et vise, très exactement, du côté oriental, le point où, à cette époque, se levait le soleil au solstice d'hiver ${ }^{2}$.

D'autres éléments architecturaux du site sont, eux, orientés nord-sud. Pourtant, les directions qu'ils adoptent ne correspondent pas simplement à la perpendiculaire à l'axe est-ouest $\left(\mathbf{2 0 6}^{\circ} \mathbf{4 3}^{\prime}\right.$ 7,35") comme on aurait pu s'y attendre. Leur décalage par rapport à cette dernière orientation est sensible et s'effectue toujours dans la même direction - vers l'est - , quoique selon un angle plus ou moins grand.

\section{Le cas de l'axe du socle de naos en quartzite de l'Akhmenou}

Il ne fait aucun doute que ce décalage est intentionnel: il est, en effet, repérable sur le socle en quartzite du sanctuaire retiré d'Amon dans l'Akhmenou ${ }^{3}$, socle qui était et est encore en grande partie inclus dans la maçonnerie de l'extrémité nord de la salle et ne peut donc avoir bougé avec le temps. L'axe du naos faisait là une angle de près de $3^{\circ}$ avec l'axe de la salle aux huit niches sur laquelle il s'ouvre et cette différence est sensible même à l'oeil nu. Les mesures exactes donnent:

\begin{tabular}{|c|c|c|}
\hline $\begin{array}{l}\text { Axe du sanctuaire de l'Akhmenou } \\
\text { (salle des huit niches) }\end{array}$ & $\begin{array}{l}\text { Axe du naos de quartzite de } \\
\text { l'Akhmenou }\end{array}$ & Différence d'azimut des deux axes \\
\hline $207^{\circ} 17^{\prime} 31,44^{\prime \prime}$ & $204^{\circ} 17^{\prime} 42,57^{\prime \prime}$ & $2^{\circ} 59 ' 48,87^{\prime \prime}$ \\
\hline
\end{tabular}

\section{Le cas de l'axe processionnel nord-sud}

Par ailleurs, la direction nord-sud de l'axe processionnel thoutmoside qui part vers Louxor est aujourd'hui encore marquée par les VII ${ }^{e}$ et VIII ${ }^{e}$ pylônes et correspond à un azimut décalé par rapport à la perpendiculaire à l'axe majeur, décalage dont rend compte le tableau suivant:

\begin{tabular}{|c|c|c|}
\hline $\begin{array}{l}\text { Axe processionnel nord-sud des } \\
\text { VIII }{ }^{\text {eVIIII }} \text { pylônes }\end{array}$ & Perpendiculaire à l'axe majeur & $\begin{array}{l}\text { Différence d'azimut des deux } \\
\text { axes }\end{array}$ \\
\hline $205^{\circ} 54 ' 39,73 \prime$ & $206^{\circ} 43^{\prime} 7,35^{\prime \prime}$ & $0^{\circ} 48^{\prime} 27,62 ”$ \\
\hline
\end{tabular}

1 J'ai évoqué ces interventions d'Antef II, de Sésostris Ier bien sûr, et de Thoutmosis III dans Le "grand château d'Amon» de Sésostris Ier à Karnak (MAIBL 17), 1998, \$179, 182-194, 220-221.

${ }^{2}$ Ibid. §201-205.

${ }^{3}$ J. Lauffray, Karnak II (= Kêmi 19), 1969, p. 201 et fig. 9. 


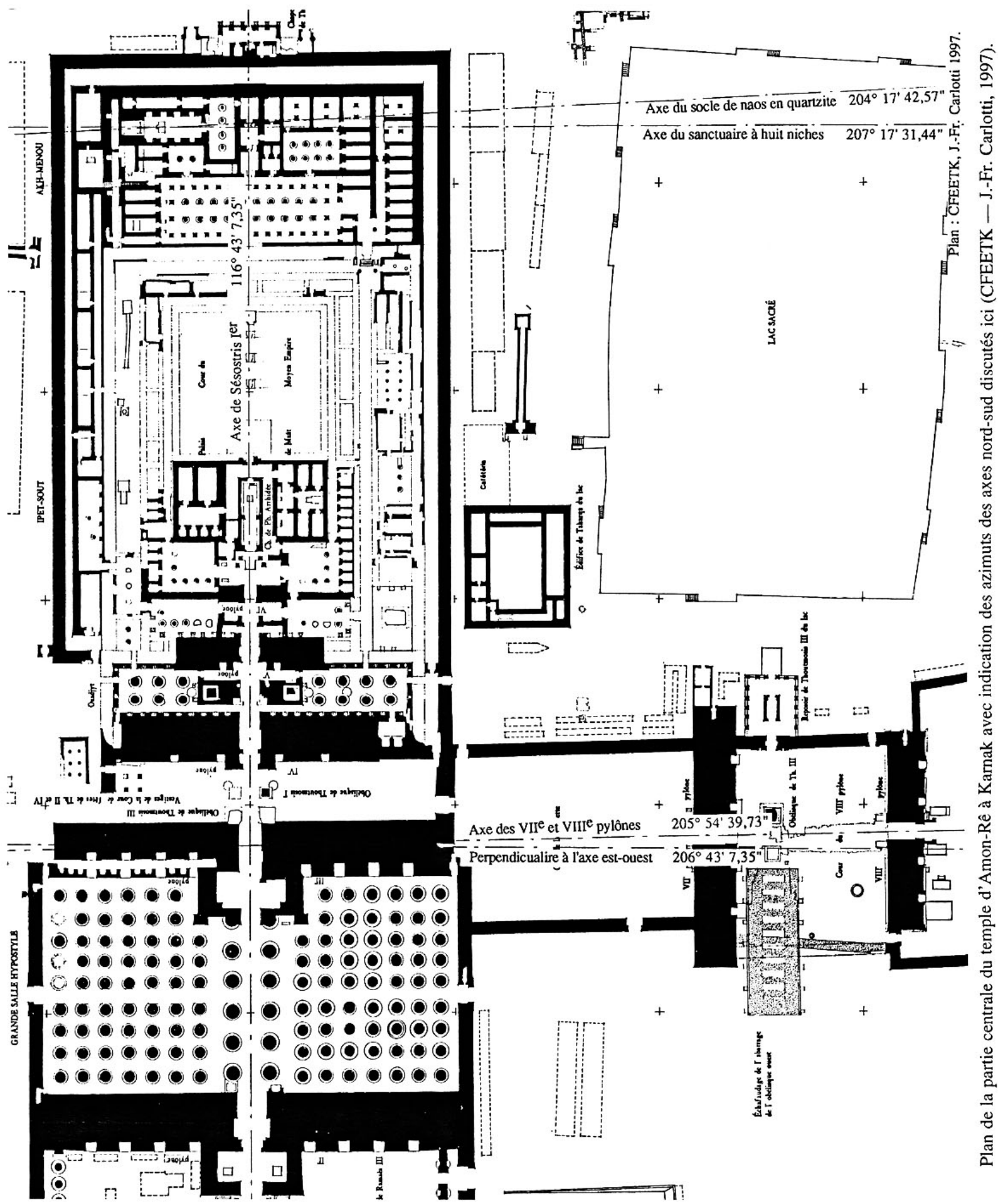

$R d E 50$ (1999) 
Cette fois-ci, le décalage vers l'est par rapport à la perpendiculaire à l'axe majeur est moindre mais il ne peut être tenu pour négligeable et est certainement volontaire.

\section{Point où se couche Canope}

Le point où se couche Canope, la seconde étoile la plus brillante du ciel après Sirius, varie dans le temps en raison du phénomène de la précession des équinoxes laquelle est conséquence de la variation de l'inclinaison et de l'orientation de l'axe de la terre sur l'écliptique. Compte tenu de la latitude et de l'altitude de Karnak, de la hauteur de l'horizon sud et de la précession des équinoxes, on peut dresser le tableau suivant de la position de cet astre au couchant ${ }^{4}$ :

\begin{tabular}{|c|c|c|}
\hline $\begin{array}{l}\text { Azimut du point où se couche } \\
\text { Canope sous Antef II }\end{array}$ & $\begin{array}{l}\text { Azimut du point où se couche } \\
\text { Canope sous Sésostris Ier }\end{array}$ & $\begin{array}{l}\text { Azimut du point où se couche } \\
\text { Canope sous Thoutmosis III }\end{array}$ \\
\hline $204^{\circ} 26^{\prime} 22,65^{\prime \prime}$ & $204^{\circ} 41^{\prime} 54,49^{\prime \prime}$ & $205^{\circ} 48^{\prime} 25,945^{\prime \prime}$ \\
\hline
\end{tabular}

Il y a parmi tous ces chiffres des convergences intéressantes qui, compte tenu de l'extrême faiblesse des différences d'angles obtenues, ne peuvent être dues au hasard. La première est la suivante:

\begin{tabular}{|c|c|c|}
\hline $\begin{array}{c}\text { Azimut de l'axe processionnel } \\
\text { nord-sud }\end{array}$ & $\begin{array}{c}\text { Azimut du point où se couche } \\
\text { Canope sous Thoutmosis III }\end{array}$ & Différence \\
\hline $\mathbf{2 0 5}^{\circ} \mathbf{5 4} \mathbf{3}^{\prime} \mathbf{3 9 , 7 3} \mathbf{y}^{\prime}$ & $\mathbf{2 0 5}^{\circ} \mathbf{4 8} \mathbf{4 8}^{\prime} \mathbf{2 5 , 9 4 5}$ & $\mathbf{0}^{\circ} \mathbf{6}^{\prime} \mathbf{1 3 , 7 8 5}$ ' \\
\hline
\end{tabular}

En se fondant sur cette forte concordance, on peut, me semble-t-il, postuler que l'axe nord-sud fut aligné sous les règnes d'Hatchepsout et de Thoutmosis III, à partir d'une visée astronomique, sur le couchant de Canope.

Le socle de naos en quartzite de l'Akhmenou a été, lui, enchâssé dans le bâtiment de Thoutmosis III. Par conséquent, il était sans doute entouré de murs et d'enceintes élevés. On ne pouvait donc procéder à une visée astronomique directe pour l'orienter. Il fallut probablement se résoudre à reprendre un azimut plus ancien. Une solution satisfaisante serait d'admettre la reprise d'une orientation remontant à l'origine du temple, selon les données suivantes:

\begin{tabular}{|c|c|c|}
\hline $\begin{array}{c}\text { Azimut du socle de naos en } \\
\text { quartzite de l'Akhmenou }\end{array}$ & $\begin{array}{c}\text { Azimut du point où se couche } \\
\text { Canope sous Antef II }\end{array}$ & Différence \\
\hline $\mathbf{2 0 4}^{\circ} \mathbf{1 7} \mathbf{1}^{\prime} \mathbf{4 2 , 5 7}$ & $\mathbf{2 0 4}^{\circ} \mathbf{2 6} \mathbf{2}^{\prime} \mathbf{2 2 , 6 5}$ & $\mathbf{0}^{\circ} \mathbf{8}^{\prime} \mathbf{4 0 , 8} \mathbf{4}^{\prime}$ \\
\hline
\end{tabular}

L'hypothèse de l'alignement sur un azimut plus ancien n'est pas une simple commodité de raisonnement indémontrable mais au contraire une éventualité rendue tout à fait vraisemblable par un parallèle qui a laissé sa trace à Karnak même. En effet, le socle de naos en calcite au nom de Sésos-

${ }^{4}$ M. P. Bretagnon du Bureau des Longitudes a eu l'amabilité de me fournir les équations qui permettent d'obtenir ces résultats et de contrôler mes calculs. Les équations sont les suivantes: a (azimut) = ArcTan (Sin $\phi^{*}$ Tan $H$ ), avec: $H$ (angle horaire $)=\operatorname{ArCos}\left[\left(\operatorname{Sin} h^{\circ}-\operatorname{Sin} \phi^{*} \operatorname{Sin} \delta\right) / \operatorname{Cos} \phi^{*} \operatorname{Cos} \delta\right] ; h^{\circ}=$ hauteur horizon $=\mathrm{P}-\mathrm{R}+\mathrm{h} ; \mathrm{P}=$ parallaxe de Canope $=$ $0,03298^{\prime \prime} ; \mathrm{R}=$ réfraction $=26^{\prime} 37^{\prime \prime}$ pour un angle de l'horizon de $\mathrm{h}=0^{\circ} 52$ ' 18,22 "; $\phi=$ latitude de Karnak $=25^{\circ} 43^{\prime} 4^{\prime \prime}$; $\delta=$ déclinaison de Canope $=54^{\circ} 43^{\prime} 12^{\prime \prime}$ sous Antef II, 54 $32^{\prime} 17^{\prime}$ 'sous Sésostris Ier à la date de refondation du temple, $53^{\circ} 53^{\prime} 28^{\prime \prime}$ sous Thoutmosis III à la date de fondation de l'Akhmenou. 
tris Ier qui fut retrouvé brisé dans et aux abords de la cour du Moyen Empire montre aujourd'hui, lui aussi, une claire dissymétrie de ses axes longitudinaux, avec un décalage entre l'axe du socle et l'axe du naos allant encore dans le même sens (vers l'est), comme s'il avait à son tour été orienté nord-sud et aligné sur une direction différente de celle des murs nord-sud du reste de l'édifice où il se trouvait 5 .

Aussi considérerai-je comme plausible que le naos de l'Akhmenou ait repris, par translation, l'azimut du point où se couchait Canope au Moyen Empire - peut-être sous Antef II, puisque c'est le plus ancien nom royal découvert à ce jour sur le site - azimut qu'avait déjà, semble-t-il, repris Sésostris Ierb.

À la question de savoir pourquoi Canope aurait été choisie à Karnak pour les alignements nordsud il ne peut pas y avoir, en l'absence de texte explicite retrouvé sur le site, de réponse certaine; mais il est possible de formuler une hypothèse. Si l'axe est-ouest rappelle sans conteste possible la course diurne du soleil et la composante solaire et héliopolitaine d'Amon-Rê, il se pourrait que l'axe nord-sud en représente l'aspect osirien et chthonien. La présence de salles sokariennes dans l'Akhmenou, d'une part, et l'ancienneté apparente des rituels de Khoiak, d'autre part ${ }^{7}$, permettent de penser que les cultes chthoniens sont apparus très tôt à Karnak. Les chapelles osiriennes des divines adoratrices autant que l'Édifice de Taharqa du lac montrent encore combien sont intimement imbriqués les cultes amonien et osirien dans ces oratoires. Les sources tardives placent enfin à Karnak même la naissance d'Osiris et, occasionnellement, assimilent ce dernier à Amon $^{8}$.

Par ailleurs, Canope, dont l'éclat est prééminent dans le ciel du sud, paraît bien avoir été mentionnée comme «Osiris-ihy», l' «étoile visible» du «ciel d'Or», sur le zodiaque de Dendara', dans un texte qui énumère les formes célestes d'Osiris, ce qui rejoint le témoignage de Plutarque selon qui Canope était étroitement associée à ce dieu:

«Ils désignent encore Osiris sous le nom de stratège, et Canopos, d'où l'astre ainsi appelé tira son nom, disent-ils, sous celui de pilote. Bien plus, ils prétendent que la nef appelée Argo par les grecs est une imitation de la barque d'Osiris et que par honneur pour ce dieu elle a été placée parmi les astres et portée non loin d'Orion et de la canicule, deux constellations que les égyptiens regardent, la première comme consacrée à Horus $\left(\right.$ sic) et la seconde à Isis.» ${ }^{10}$

\footnotetext{
${ }^{5}$ Pour l'orientation nord-sud vraisemblable de ce socle de naos, voir Karnak X, 1995, p. 253-256.

${ }^{6}$ Pour J.N. Lockyers (The Dawn of Astronomy, 1894, p. 184, 185, 186 et surtout 310) le temple d'Aménophis III à Karnak nord, le reposoir de Séthi II à Karnak, le temple de Khonsou auraient été orientés sur le point où se couchait Canope. Ses mesures des axes sont trop anciennes pour être fiables et on ne peut plus suivre ses conclusions historiques.

${ }^{7}$ Des enterrements de figurines osiriennes et sokariennes pourraient remonter au Nouvel Empire et être antérieures, en tout cas, à la XXI' ${ }^{\mathrm{e}}$ dynastie. J. Leclant, Orientalia 65/3 (1994-1995), p. 270; Fr. Leclère, Egyptian Archeology 9 (1996), p. 9-12; Fr. Leclère, L. Coulon Actes du VIIe Congrès international des égyptologues de Cambridge (OLA 82), 1998, p. 651.

${ }^{8}$ Amon-Rê-Ounennefer: C. de Wit, Les inscriptions du temple d'Opet d Karnak (BiAe XI), 1958, p. 90 et 110; N. de G. Davies, The Temple of Hibis III, 1953, pl. 7, jambage gauche de la porte de gauche; Amon-Rê qualifié de «Ba vénérable d'Osiris», C. de Wit, op. cit., p. 91, 121, cf. aussi, R.A. Parker, J. Leclant, J.-Cl. Goyon, The Edifice of Taharqa, 1979, p. 78; ou encore «Rê-Amon, que l'on appelle Osiris», C. de Wit, op. cit., p. 233, 1. 5.

9 S. Cauville, Dendara. Les chapelles osiriennes. Texte 175; Traduction et transcription (BdE 117), 1997, p. 90; Commentaires ( $B d E 118), 1997$, p. 79-80, et surtout, pour l'identification de l'étoile, la démonstration donnée dans E. Aubourg, S. Cauville dans In memoriam J. Quaegebeur II (OLA 85), 1998, p. 767-772.

${ }^{10}$ Plutarque, Isis et Osiris chapitre 22 (=359 E) et commentaire de J.G. Griffiths, Plutarch's de Iside et Osiride, 1970, p. $376-377$.
} 
Les orientations données aux plus importants éléments architecturaux du site de Karnak témoigneraient alors des équivalences théologiques suivantes:

- axe est-ouest,

visée sur le point où se lève le soleil au solstice d'hiver $>$ Rê $>$ aspect solaire d'Amon;

- axe nord-sud,

visée sur le couchant de Canope $>$ Osiris $>$ aspect chthonien d'Amon.

Résumé / Abstract

Les axes nord-sud de Karnak, tels qu'ils sont repérables sur le socle du sanctuaire de quartzite de l'Akhmenou ou sur l'axe processionnel des VII et $\mathrm{VIII}$ e pylônes, présentent des décalages vers l'est. Ceux-ci paraissent coïncider avec l'azimut du couchant de Canope sous Antef II pour l'un et sous Thoutmosis III pour l'autre. La direction nord-sud serait alors à mettre en relation avec l'aspect chthonien de Canope (assimilée à Osiris), opposé à la dimension solaire de l'axe est-ouest.

The north-south axis of Karnak, as they can be established from the quartzite naos basis of the Akhmenu or from the procession axis of the VIIth and VIIIth pylons, show shifts to the east. These shifts seem to fit with the azimut of the setting Canopus under the reign of Antef IInd for the first and under the reign of Thutmosis IIIrd for the second. The nord-south orientation should then be related with the chthonian aspect of Canopus (assimilated to Osiris), opposed to the solar aspect of the east-west axis.

Luc GABOLDE

Mission du CNRS à Karnak

Ambassade de France au Caire

128bis, rue de l'Université

F-75351 PARIS Cedex 07 